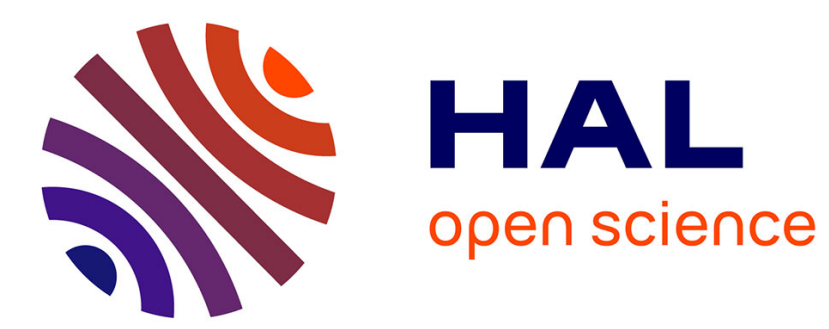

\title{
Victor Hugo entre réception patrimoniale et réception politique (1902-1944)
}

\author{
Jordi Brahamcha-Marin
}

\section{To cite this version:}

Jordi Brahamcha-Marin. Victor Hugo entre réception patrimoniale et réception politique (1902-1944).

Culture et Musées, 2021, 38, pp.29 - 50. 10.4000/culturemusees.6592 . hal-03471472

\section{HAL Id: hal-03471472 \\ https://hal.science/hal-03471472}

Submitted on 8 Dec 2021

HAL is a multi-disciplinary open access archive for the deposit and dissemination of scientific research documents, whether they are published or not. The documents may come from teaching and research institutions in France or abroad, or from public or private research centers.
L'archive ouverte pluridisciplinaire HAL, est destinée au dépôt et à la diffusion de documents scientifiques de niveau recherche, publiés ou non, émanant des établissements d'enseignement et de recherche français ou étrangers, des laboratoires publics ou privés. 


\section{OpenEdition}

Journals

\section{Culture \& Musées}

Muséologie et recherches sur la culture

$38 \mid 2021$

Patrimonialisations de la littérature

Dossier

\section{Victor Hugo entre réception patrimoniale et réception politique (1902-1944)}

Victor Hugo between heritage and political receptions (1902-1944)

\section{JORDI BRAHAMCHA-MARIN}

p. $29-50$

https://doi.org/10.4000/culturemusees.6592

\section{Résumés}

Français English Español

Le statut de Victor Hugo comme auteur du patrimoine entre 1902 et la fin de la Seconde Guerre mondiale semble aussi évident qu'instable. Honoré par l'école, statufié dans maints lieux publics, désigné comme " notre grand poète » ou " notre grand Victor Hugo », il jouit apparemment d'une gloire consensuelle. Mais il appartient à un passé trop récent pour que ces hommages ne soient pas contestés : la gauche en général, et le Parti communiste en particulier, tentent de l'arracher au consensus républicain pour en faire une réception plus activement politique ; tandis que l'Action française, à l'extrême droite, dénonce ces célébrations comme une forme insidieuse de militantisme républicain. La figure de Hugo est donc écartelée entre une réception patrimoniale et une réception politique. Cette opposition s'estompe pendant la Résistance, puisque dans un contexte où l'existence même de la patrie est menacée, la célébration du patrimoine est en elle-même une arme politique.

Victor Hugo's belonging to cultural heritage between 1902 and the end of the Second World War seems both obvious and precarious. Honoured by the educational institution, immortalized in the form of a statue in many public places, frequently referred to as "our great poet" or "our great Victor Hugo", he apparently enjoys a consensual glory. But he belongs to a past too recent for these tributes not to be challenged; the left in general and the Communist Party in particular try to wrest him from the republican consensus and to actively promote a more political reception, whereas the far-right Action française denounces these celebrations as an insidious form of republican militancy. Hugo is thus split between his reception as a heritage figure, and a political reception. This opposition faded away during the Resistance: in a context where the very existence of the fatherland is threatened, the celebration of heritage is by itself a political weapon. 
La condición de autor del patrimonio que posee Víctor Hugo entre 1902 y el final de la Segunda Guerra Mundial parece tan evidente como inestable. Honrado por la academia, perpetuado en muchos lugares públicos, llamado "nuestro gran poeta" o "nuestro gran Víctor Hugo", el autor goza aparentemente de una gloria generalizada. Sin embargo, pertenece a un pasado demasiado reciente para que estos homenajes no sean cuestionados: la izquierda en general, y el Partido Comunista en particular, intentan desprenderlos del consenso republicano, para que su recepción sea más activamente política, mientras que el movimiento de ultraderecha Action Française denuncia estas celebraciones como una forma insidiosa de militancia republicana. Es así que la figura de Hugo se divide entre una recepción patrimonial y una recepción política. Esta oposición se atenuó durante la Resistencia, ya que en un contexto en el que la propia existencia de la patria se encontraba amenazada, la celebración del patrimonio era en sí misma un arma política.

\section{Entrées d'index}

Mots-clés : patrimoine, Hugo (Victor), République, Parti communiste, Action française, Résistance

Keywords: heritage, Hugo (Victor), Republic, Communist Party, Action française, Resistance Palabras clave: patrimonio, Hugo (Víctor), República, Partido Comunista, Action française, Resistencia

\section{Notes de la rédaction}

Manuscrit reçu le 6 janvier 2021

Version révisée reçue le 10 mai 2021

Article accepté pour publication le 28 juin 2021

\section{Texte intégral}

Vu le nombre de rues et d'écoles qui portent son nom (Martinet, $1985: 262-270$ ), il paraît évident que les pouvoirs publics, tout au long du xxe siècle, ont eu à cœur de rendre hommage à celui qui passe régulièrement pour « le plus grand poète français ». " La gloire de Victor Hugo », pour emprunter son titre à l'exposition organisée au Grand Palais en 1985-1986, semble aller de soi : à première vue, la référence à Hugo est suffisamment consensuelle, la diffusion scolaire de son œuvre suffisamment large, pour que l'auteur des Contemplations et des Misérables puisse être considéré, de manière non problématique, comme appartenant au patrimoine culturel commun des Français. Pour autant, ce consensus n'a de cesse d'être contesté, sur ses marges, par des discours qui semblent souvent surestimer leur propre nouveauté, qui semblent oublier ou ignorer les longues et puissantes traditions critiques auxquelles ils se rattachent. On croit, par exemple, être le premier ou presque à s'en prendre à Hugo ; on croit faire preuve d'un iconoclasme sans précédent en dénonçant sa démagogie, son orgueil ou quelque autre vice moral ; mais ce faisant, l'on méconnait que l'« hugophobie » est aussi vieille que Hugo lui-même et qu'elle fut portée voici un siècle par des forces politiques puissantes, l'Action française principalement. Tout se passe comme s'il y avait autour de la figure de Hugo davantage qu'un véritable consensus, un fantasme de consensus, autrement dit comme si nombre de commentateurs avaient le sentiment un peu vague, pas toujours bien fondé et parfois agacé ou angoissé, d'évoluer dans un univers culturel acquis à la cause de Victor Hugo. Au lendemain du bicentenaire de la naissance du poète, en 2002, Claude Millet écrit ainsi ces quelques phrases suggestives pour résumer l'histoire de la réception du poète :

\footnotetext{
« C'est une gloire toujours inscrite dans les polémiques du temps, et la lecture, la réception de Hugo s'est toujours greffée sur ces polémiques. Plutôt que le poète de la France, Hugo est son réactif. Hugo n'a donc jamais été, et n'est pas une figure de rassemblement, fondatrice de la nation française dans son unanimité. En ce sens, ce grand-homme-de-la-patrie n'est pas un héros épique mais le théâtre d'un conflit dramatique, celui des divisions de la nation, travaillée par la contradiction des opinions (Millet, 2002-2003 : 8). »
}

Hugo a toujours été une figure clivante et une figure contestée ; mais aussi bien ceux qui l'ont promue que ceux qui l'ont contestée ont fréquemment entretenu à son sujet le mythe d'une figure plus consensuelle qu'elle ne l'était réellement. En quelque sorte, 
l'idée d'un Hugo " grand-homme-de-la-patrie », père de la nation et garant posthume de son unité fait autant partie de la légende hugolienne que d'autres mythèmes biographiques comme l'image du vieux républicain exilé sur son rocher ou celle du vieillard barbu attendri par ses petits-enfants ; mais elle est peut-être un peu plus fausse qu'elle n'en a l'air. Le consensus autour de Hugo n'est pas un fait objectif observable dans sa réception critique, mais un fantasme que les discours critiques produisent à propos des autres discours auxquels ils répondent et auxquels ils s'adressent.

3 Il ne s'agit évidemment pas de nier qu'il y ait eu une patrimonialisation de la figure de Hugo ; nous allons, dans un premier temps, nous attacher à en montrer quelques traces. Mais la chose délicate est de distinguer ce qui relève, dans ce processus de patrimonialisation et de célébration, d'une gloire acquise et ce qui relève, au contraire, d'un discours volontariste visant à instaurer performativement ce même consensus. Aussi verrons-nous quelques-unes des tensions qui s'instaurent entre une réception patrimoniale et une réception politique de Hugo, la seconde minant les aspirations qui fondent la première.

4 Cet article se concentre sur la première moitié du xxe siècle ; son empan couvre la période qui va de 1902 (centenaire de la naissance du poète) jusqu'à la fin de la Seconde Guerre mondiale. Figure contestée de son vivant, cible d'attaques très virulentes de la part des auteurs réactionnaires qui ne lui pardonnent ni son engagement républicain ni sa sympathie pour les communards réprimés, Hugo avait irrité à gauche : le pamphlet de Paul Lafargue sur La Légende de Victor Hugo, paru (assez confidentiellement) en 1885, témoigne d'une certaine hugophobie socialiste - mais celle-ci n'est guère influente, et surtout vivace, semble-t-il, dans le courant guesdiste (Lafargue, 1979 : 3133). Ce sont plutôt en général des auteurs de droite (Edmond Biré, Louis Veuillot...) qui accablent Hugo. La date de 1902 constitue un jalon important pour les phénomènes qui vont nous occuper ; certains indices matériels suggèrent qu'il y a à cette occasion une accélération du processus de patrimonialisation de Hugo (c'est à partir de cette date qu'on érige massivement des statues à son effigie, et c'est en 1903 que l'on ouvre un musée qui lui est consacré). En fait, au début du xxe siècle, la République semble complètement installée, après avoir survécu à la crise boulangiste et à l'affaire Dreyfus ; les grandes figures qui lui sont associées peuvent désormais acquérir le statut d'icônes. La réception de Hugo cesse d'être principalement militante pour devenir, précisément, patrimoniale ; et c'est justement en réaction à ce processus de patrimonialisation qu'une repolitisation de la figure de Hugo peut avoir lieu. Ainsi, les héritiers idéologiques de Biré ou Veuillot au xxe siècle diffèrent de leurs ancêtres par ceci que leur hugophobie ne s'inscrit plus dans le cadre d'un affrontement entre république et monarchie, entre progrès et réaction, mais se donne désormais pour une idéologie de vaincus, se perçoit comme une passion minoritaire aux prises avec un consensus (républicain et hugophile) écrasant. Quant à la Seconde Guerre mondiale, elle correspond à une période où l'opposition entre logique patrimoniale et logique politique est redéfinie, voire annulée.

\section{Victor Hugo, écrivain patrimonial}

Commençons par relever un signe discret mais significatif de la «patrimonialisation » de Victor Hugo dans la première moitié du xxe siècle. Bon nombre d'auteurs ont tendance à le désigner comme " notre poète ", " notre grand lyrique », etc. : le choix d'un déterminant possessif n'est pas innocent. Hugo est ainsi «notre poète national » dans un entrefilet non signé du Petit Parisien, le 18 mars 1915 (Le Petit Parisien, 1915 : 2) ; il est " notre grand Hugo » dans Le Journal du 13 décembre 1915 (Duguet, 1915 : 5), dans Le Populaire du 19 août 1930 (Lermina, 1930 : 4) ou dans Le Petit Parisien du 26 octobre 1935 (là, « notre grand Hugo » voisine avec « notre Lamartine » et « notre Mérimée » qui, eux, ne sont pas « grand[s] »; Le Petit Parisien, 1935 : 7). À la Chambre des députés, le républicain-socialiste Frédéric Brunet, en 1919, invoque « notre grand poète national » à l'appui de son discours 
pacifiste (Journal officiel, 1919 : 2014), et son collègue du centre droit Gratien Candace défend en 1924 la corporation des pêcheurs, ces « travailleurs de la mer " jadis glorifiés par « notre grand poète Victor Hugo » (Journal officiel, 1924 : 3509). Relevons aussi que Paul Claudel, en 1935, à l'occasion des commémorations du cinquantenaire de la mort de Hugo, se retrouve invité à louer « notre grand poète national », ce qu'il fera par un discours prononcé à Bruxelles (cité dans Antoine, 2002 : 239). Toutes ces expressions révèlent à la fois un rapport affectif à l'écrivain dont on parle, la volonté d'établir une connivence avec les destinataires du discours ou du propos et, donc, le sentiment d'une propriété commune (nationale) sur Hugo et son œuvre.

6 Concrètement, comment se manifeste ce rapport patrimonial à Hugo dans la période que nous étudions ? Il prend tout d'abord, principalement à partir du centenaire de 1902 (Martinet, 1985 : 272), la forme d'une présence ostensible dans l'espace public par l'érection de statues. En 1902, année du centenaire de sa naissance, Hugo est statufié par le sculpteur Barrias à Paris : son effigie en bronze trône désormais sur la place qui porte son nom jusqu'en 1941, date où elle est déboulonnée et fondue par les Allemands, le bronze devant être réutilisé dans l'industrie. Ce choix de déboulonner Hugo, comme d'autres grandes figures de la IIIe République, est naturellement très politique (Hargrove, 1997 : 1879). Dans un cas comme celui-ci, l'affront fait au grand homme est redoublé par un affront fait à l'œuvre d'art, au monument public, ce qui contrevient frontalement à une logique de préservation patrimoniale des biens artistiques. Barrias n'est pas le seul sculpteur à installer Hugo dans l'espace public ; quelques semaines avant la Grande Guerre, c'est à Guernesey, son lieu d'exil, qu'un Hugo de granit, réalisé par Jean Boucher, est inauguré ; la Nation et l'Académie françaises sont représentées par Jean Richepin (Richepin, 1914).

Les statues de Hugo ne sont pas seulement des monuments matériels posés dans les villes ou sur les îles, elles sont aussi des objets textuels. En 1925, dans ses « Réflexions et propositions sur le vers français ", Claudel consacre de belles pages (Claudel, 1965 : 19-22) au marbre de Hugo par Rodin conservé à la Glyptothèque de Copenhague. Dans " Langage des statues (fragment) », poème écrit par Aragon en 1942 et recueilli en 1943 dans En français dans le texte, puis en 1945 dans En étrange pays dans mon pays luimême, c'est la statue de Hugo par Barrias qui descend de son socle pour stigmatiser l'hermétisme des surréalistes (Aragon, 2007 : 906-908)1. Aragon, grand lecteur de Hugo, fait donc parler celui-ci sous la forme d'une statue ; le poème prend acte de la patrimonialisation de Hugo et la réitère au sein de son poème : Hugo se confond avec les monuments érigés à sa gloire. La conscience de ce statut patrimonial de Hugo est tout aussi vive chez ceux qui s'en agacent. Ainsi, un numéro du Surréalisme au service de la révolution, en mai 1933, demande aux membres du groupe surréaliste si l'on doit, selon eux, « conserver, déplacer, modifier, transformer ou supprimer » 31 monuments parisiens énumérés (Le Surréalisme au service de la Révolution, 1933:18), or la statue en bronze de Hugo par Rodin, installée dans les jardins du Palais-Royal en 1909, est le 28e monument de la liste. Quelques réponses témoignent d'un iconoclasme antihugolien : Tristan Tzara propose de remplacer la statue « par un amplificateur puissant branché sur l'Observatoire et hurlant l'heure de dix secondes en dix secondes » (ibid. : 19 ; variation sur le cliché d'un Hugo sonore, voire bruyant), et Paul Éluard de l'« entourer d'un groupe de petits enfants très habillés et copulant entre eux » (ibid. : 18 ; double allusion à la poésie de Hugo sur les enfants, souvent suspectée de fadeur, et à la vitalité sexuelle légendaire du poète ${ }^{2}$ ). Tzara et Éluard sont, depuis le début, parmi les moins hugophiles du groupe surréaliste (contrairement à Aragon, Breton ou Desnos : voir Brahamcha-Marin, 2018 : 677-690), et il n'est pas indifférent qu'ils profitent de l'occasion que leur donne cette enquête pour s'en prendre à un signe visible de la patrimonialisation de Hugo : non seulement à Hugo, donc, mais à Hugo en tant qu'il est monumentalisé, statufié.

8 De même, quantité de rues, avenues, places ou boulevards reçoivent le nom de l'auteur des Misérables ; l'appartement de Hugo, place des Vosges, est transformé en musée en 1903. Au lendemain de la Grande Guerre, des vers d'un poème de Hugo ( "Hymne », tiré du recueil Les Chants du crépuscule de 1835) sont souvent gravés sur les monuments aux morts. La référence hugolienne condense la plupart des connotations sémantiques étudiées par Antoine Prost (1997) à propos des monuments 
aux morts : elle articule harmonieusement une dimension patriotique et républicaine, contribuant rétrospectivement à une relecture de la Grande Guerre comme guerre patriotique et comme guerre de défense républicaine. C'est en tout cas sur Hugo que l'on compte pour cimenter, dans l'épreuve du deuil collectif, le sentiment national - et pour appuyer ce sentiment national sur la mémoire des guerres et des révolutions passées, en l'occurrence les Trois Glorieuses de juillet 1830 à propos desquelles le poème de Hugo a été écrit (Brahamcha-Marin, 2017).

9 L'État, les pouvoirs publics et différentes institutions culturelles prennent donc en charge l'entretien de la mémoire hugolienne, à la fois par l'installation durable de traces hugoliennes dans l'espace public et par des célébrations plus ponctuelles à l'occasion de dates clés : 1902 (le centenaire de la naissance), 1935 (le cinquantenaire de la mort), mais aussi 1927 (le centenaire de Cromwell et de sa préface) et 1930 (le centenaire d'Hernani et de sa bataille), ces deux dernières dates valant, en vertu d'une association métonymique entre Hugo et le romantisme, comme anniversaires du romantisme. La Comédie-Française et la Bibliothèque nationale participent activement, sous pilotage ministériel, auxdites célébrations (Brahamcha-Marin, 2018 : 772-781). Les commémorations de 1935 sont à la fois les plus nombreuses et les plus polémiques, car la gauche, communistes en tête, reproche au gouvernement la tiédeur avec laquelle il célèbre le grand homme. Discours, expositions, représentations théâtrales ont cependant bien lieu ; les pouvoirs publics suscitent, fût-ce parfois avec un soupçon de réticence, un hommage à Hugo comme grande figure nationale.

Reste à parler de l'école, qui est, comme Pierre Nora l'a suggéré, le principal lieu d'élaboration et de conservation patrimoniale sous la IIIe République (Nora, 2011 : 105). Hugo fait une entrée dans les programmes et les manuels scolaires à la fin du xixe siècle, puis, progressivement, dans les sujets de baccalauréat. La littérature romantique en général, souvent résumée, au moins pour la poésie, par le quatuor Hugo-Lamartine-Musset-Vigny, gagne progressivement du terrain par rapport à la littérature de l'âge classique, mais Hugo surclasse en général, quantitativement parlant, ses trois compagnons (Brahamcha-Marin, 2018 : 46-71 et 91-93). Sans entrer ici dans le détail des textes sélectionnés par les manuels, relevons que le retour insistant de quelques grands classiques - "Oceano nox », tiré des Rayons et les Ombres, 1840 ; "L'expiation », tirée de Châtiments, 1853 ; "Saison des semailles. Le soir », tirée des Chansons des rues et des bois, 1865 ; "Jeanne était au pain sec... », tiré de L'Art d'être grand-père, 1877 ; ainsi que, surtout en primaire, quelques extraits des Misérables, parus en 1862 (Brahamcha-Marin, $2018: 58-71$ ) - permet l'appropriation par tous les élèves de France de quelques « lieux de mémoire».

Hugo n'est certes pas le seul auteur à connaître ce processus de patrimonialisation ; de manière générale, le « grand écrivain » est dans la France de l'entre-deux-guerres une figure révérée et respectée. Des auteurs - vivants ou morts -, comme André Gide, Anatole France, Paul Bourget, Maurice Barrès, sont des figures publiques et des maîtres à penser dont l'influence va bien au-delà de tel ou tel cercle partisan : on sait, par exemple, en quelle estime Aragon tenait Barrès. Ce statut social prestigieux de la littérature favorise, naturellement, la patrimonialisation des auteurs des époques passées. Hugo n'est certes pas le seul à en bénéficier - sa fortune scolaire n'éclipse pas celle des auteurs du Grand Siècle, La Fontaine, Molière, Corneille, Racine, patrimonialisés plus tôt et plus durablement que lui -, mais il est celui dont la réception s'articule le plus étroitement avec l'histoire de la République.

\section{Réception patrimoniale contre réception politique}

Ce rapide tableau pourrait suggérer que le processus de patrimonialisation de Hugo est très naturel et très harmonieux ; or rien n'est moins vrai. Car comme Nathalie Denizot et Violaine Houdart-Mérot l'ont bien noté, la logique patrimoniale suppose en principe une rupture entre le passé patrimonialisé et le présent : la récupération mémorielle est censée combler un sentiment d'extranéité (Denizot, 2015 : 111-112 ; 
Houdart-Mérot, 2015 : 39). Voilà pourquoi, à la fin du xixe siècle, ce sont d'abord les auteurs du Grand Siècle qui se retrouvent patrimonialisés par l'école. Dans la première moitié du xxe siècle, Hugo n'est certes plus tout à fait un contemporain, mais il n'appartient pas non plus à un passé révolu : il est l'un des pères fondateurs symboliques du régime politique. Homme de la IIIe République, Hugo peut donc dès lors avoir quelque chose à dire sur la politique de la Belle Époque, de la Grande Guerre, de l'entre-deux-guerres, de la Résistance ; témoin et acteur de l'époque qui a inventé ou installé, en France, la république, le parlementarisme, le libéralisme, la « question sociale ", il est régulièrement convoqué comme allié dans un combat, comme caution d'une prise de position, comme précurseur d'une ligne politique ; et cet usage militant de Hugo l'arrache au consensus que réclame la logique patrimoniale. Chantal Martinet note d'ailleurs cette tension en constatant que la multiplication des statues de Hugo à partir de 1902 est corrélée à une dépolitisation de la figure de Hugo : la figure de Hugo " grand poète », dans les représentations statuaires, prend le pas sur la figure de Hugo " grand républicain » (Martinet, $1985: 275-276)$. La République est désormais assez bien installée pour que le républicanisme lui-même puisse être, au moins en apparence, une valeur hégémonique, voire consensuelle. Mais sur fond d'un consensus hugophile largement fantasmé, les conflits politiques reprennent leur droit.

C'est un débat récurrent, pendant tout le premier xxe siècle, que de savoir à quel point il peut y avoir un consensus autour de la figure de Hugo, et donc aussi jusqu'où le processus de patrimonialisation de Hugo peut être mené. On peut identifier une position officielle - celle du gouvernement et des partis de gouvernements (radicaux et modérés), des institutions culturelles publiques, ainsi que de beaucoup d'universitaires - qui mobilise la mémoire de Hugo comme " grand homme » et " grand poète », qui honore ses combats sociaux et humanitaires en termes généraux et qui cherche à créer autour de lui un consensus aussi large que possible. Cette position est ébranlée sur sa gauche et sur sa droite. Elle l'est sur sa gauche par ceux qui cherchent à politiser Hugo pour en faire un compagnon de route de leur propre parti, et donc qui contestent la possibilité d'une appropriation consensuelle transpartisane ; elle l'est sur sa droite par les militants, théoriciens et sympathisants de l'Action française qui rejettent aussi bien Hugo que le consensus républicain qu'il est censé incarner.

Commençons par ces attaques venues de la droite. Le cours « Victor Hugo » ouvert en Sorbonne en 1926 en fournit un exemple particulièrement intéressant. À l'origine de ce cours se trouve un comité de patronage fondé en 1922 puis transformé en 1925 en Fondation Victor-Hugo ; de nombreuses notabilités en sont membres : écrivains, universitaires, mais aussi hommes politiques de premier plan, comme Édouard Herriot et Louis Barthou's. Les présidents de la République en sont présidents d'honneur. Ce « cours », qui aurait dû être une chaire magistrale si les souscriptions avaient recueilli assez d'argent pour le pérenniser, constitue donc un hommage à Hugo rendu à la fois par l'Université et la République. Les études littéraires scientifiques se mettent donc au service d'un projet de glorification de Hugo - et, à travers lui, de la République. Si les leçons elles-mêmes (dispensées successivement, jusqu'à la guerre, par le poète Fernand Gregh puis les universitaires André Le Breton et Georges Ascoli) s'adressent à un auditoire restreint, il faut noter que le comité de patronage a initialement envisagé d'associer largement, via des souscriptions, les citoyens et même les enfants des écoles à ce projet (Le Temps, 1922*). L'historien Jules Bertaut relève qu'il s'agit bien d'un " hommage national » (ibid.), et, de fait, les modalités de mise en œuvre de ce projet cherchent à créer les conditions d'une appropriation de la référence hugolienne par une partie large de la nation.

15 Or la création de ce cours donne lieu à de virulentes critiques émanant de l'Action française et de ses sympathisants. L'extrême droite maurrassienne est violemment antihugolienne et antiromantique, par antirépublicanisme et antigermanisme ; la thèse polémique de Pierre Lasserre, en 1907, sur (et contre) Le Romantisme français constitue l'un des premiers grands jalons de cette campagne. Léon Daudet, l'un des idéologues du mouvement (et par ailleurs ancien époux de la petite-fille de Hugo), publie en 1922 un pamphlet intitulé Le Stupide xıxe siècle, où le romantisme en général et Hugo en particulier font l'objet de critiques dévastatrices. En 1926, au moment de la création effective du cours, les maurrassiens protestent dans la presse (Bonnard, 
1926* ; Daudet, 1926* ; Tuc, 1926*), trop heureux de pouvoir attaquer dans le même mouvement Hugo, la République et ce temple républicain qu'est alors la Sorbonne (Chaline, 2007 : 78-79). Cet épisode survient peu de temps après une affaire qui avait déjà mis aux prises l'Action française et le monde universitaire : au printemps 1925, les étudiants maurrassiens s'étaient mobilisés contre la nomination de Georges Scelle, directeur de cabinet du ministre (radical) du Travail, comme professeur de droit public à l'université de Paris (Prévotat, 2004 : 40). C'est dans la continuité de cette précédente mobilisation que le mouvement royaliste, en 1926, s'attaque à nouveau à une institution universitaire supposée corrompue et acquise au régime en place.

16 Si l'on considère les attitudes adoptées par les défenseurs de la chaire ou du cours "Victor Hugo », on consate que la querelle de 1926 constitue une césure. Avant cette date, plusieurs auteurs, comme Henry Céard (1922*), Ernest Zyromski (1925 : 1), Gaston Arthuis (1926*) ou Fernand Nozière (1926*), voient dans cette initiative la preuve que Hugo est devenu une figure consensuelle et patrimoniale, et le moyen d'entériner sa gloire ; mais après que les maurrassiens ont engagé le combat, d'autres auteurs, comme le très hugophile critique du Temps Paul Souday (1926a* et $1926 \mathrm{~b}^{*}$ ), prennent acte du dissensus et se font fort d'y répondre en défendant Hugo (BrahamchaMarin, 2018 : 301-306). La réalité a en fait démenti le rêve consensualiste. Mais au-delà de Hugo, ce qui est menacé, c'est la République elle-même - dont Hugo est un symbole : c'est bien par antirépublicanisme que les intellectuels de l'Action française s'en prennent à l'auteur des Misérables. Et l'impossibilité d'une patrimonialisation sereine de Hugo est le révélateur, dans le champ littéraire, d'une instabilité chronique du régime républicain dans l'entre-deux-guerres.

La réception de Hugo fait aussi difficulté à gauche, car une tension s'instaure entre une référence consensualiste et patrimoniale d'une part, et une référence militante (de gauche) d'autre part. Les députés de gauche et ceux de droite, quand ils citent Hugo à la Chambre, n'en font généralement pas le même usage. L'usage mémorial ou patrimonial de la référence hugolienne se pratique sur tous les bancs - référence vague qui mobilise alors seulement l'image de Hugo comme grand homme. Mais la pratique consistant à utiliser Hugo comme caution d'une proposition ou comme argument d'autorité à l'appui d'une mesure est rare à droite, plus fréquente à gauche. En 1922, la campagne pour l'amnistie des mutins de la mer Noire, portée à la Chambre par les groupes socialiste et communiste, peut ainsi se réclamer, comme dans un discours de Jules Uhry (SFIO), d'un célèbre passage d'Hernani (1830) où l'empereur Charles Quint promet de commencer son règne " par la clémence » (Journal officiel, 1922 : 2989). En revanche, le discours de Léon Bérard (Alliance démocratique, centre droit) qui, en 1916, place Hugo au-dessus de Pascal ou de Rodin parmi les grands hommes de la République (Journal officiel, 1916 : 1803), est assez typique des discours de droite, qui se méfient trop des potentialités subversives de l'œuvre de Hugo pour aller au-delà des hommages très généraux. Bien sûr, la distinction entre des usages politiques de Hugo (à gauche) et des usages patrimoniaux (à droite) est un peu rapide et mériterait d'être affinée, notamment parce qu'il existe toute une catégorie intermédiaire d'usages semipolitiques, moraux (on invoque de manière un peu vague la tendresse de Hugo pour les enfants, ou sa sympathie pour les misérables...), qui sont moins nettement le monopole de la gauche que ses usages strictement politiques. Il n'empêche : dans les grandes lignes, on distingue, au moins sous forme de polarités, d'une part un camp qui est à l'aise avec le Hugo militant, de l'autre un camp qui reconnaît davantage en lui une figure (consensuelle) du patrimoine national. La droite n'utilise pas (ou rarement) Hugo contre la gauche, la gauche en revanche utilise bien Hugo contre la droite.

18 C'est au printemps 1935, alors que l'on célèbre le cinquantenaire de la mort du poète, que cette tension autour de Hugo et cette volonté de la gauche d'en faire un usage partisan apparaissent le plus clairement. La France est alors dirigée par un gouvernement de centre droit qui organise une série d'événements commémoratifs. Mais ceux-ci sont jugés scandaleusement indigents par la SFIO et surtout par le Parti communiste (PC), qui voit dans ce manque d'entrain gouvernemental la preuve que les partis bourgeois ne peuvent plus revendiquer Hugo : celui-ci, par son progressisme et sa sympathie pour les opprimés, ne peut plus désormais être réclamé que par le camp du prolétariat (voir, par exemple : Aragon, 1935 ; Fréville, 1935 ; L’Humanité, 1935 ; 
Nizan, 1935). Or, pour les partis du Front populaire, très actifs et très offensifs en matière culturelle, et très soucieux de mener une politique de " réintégration patrimoniale » dans une logique « unanimiste » (Ory, 1994: 842), la question de savoir ce qu'il convient de faire de Hugo n'est évidemment pas anecdotique ; elle l'est d'autant moins que plusieurs intellectuels communistes, socialistes, radicaux ou sympathisants de ces courants préparent à ce moment-là un Congrès mondial des écrivains pour la défense de la culture qui se tiendra du 21 au 25 juin 1935 (ibid. : 188-192)4. La question littéraire est donc d'une actualité brûlante à gauche, et Hugo bénéficie de cette conjoncture. Certains périodiques qui servent de passerelle entre les différents courants de la gauche (communiste et non communiste) communient d'ailleurs dans l'hommage à Hugo : le numéro spécial de la revue Europe en juin 1935 cimente autour de Hugo toutes les forces qui obtiendront une majorité parlementaire quelques mois plus tard sous l'étiquette du Rassemblement populaire. Sous l'impulsion de Louis Aragon et de quelques autres (dont Maurice Thorez), le PC adopte une nouvelle stratégie culturelle qui rompt avec le sectarisme des années précédentes et qui vise à cimenter les bases idéologiques de son alliance avec la SFIO et, bientôt (à partir de l'été 1935), avec le Parti radical dans le cadre du Rassemblement populaire ; la réappropriation de Hugo par les communistes constitue dès lors un enjeu stratégique 5 . Sans doute les communistes, à cet égard, font-ils d'ailleurs preuve de davantage de zèle que leurs alliés socialistes et radicaux, car ces deux derniers courants ne se considèrent plus depuis longtemps comme étant « en état d'objection de conscience à l'égard de la culture classique et nationale » (ibid. : 62) : l'hugophilie d'un Édouard Herriot, d'un Alexandre Bracke (député SFIO et cadre du parti) ou d'un Léon Blum va presque de soi et, par conséquent, ne nécessite guère une mise en scène tonitruante. Les communistes, en revanche, doivent surjouer leur ralliement aux emblèmes nationaux - Jacques Duclos, au nom du PC, et en rupture avec quinze ans d'internationalisme militant, revendique, par exemple, en 1935, le drapeau tricolore « dont nos ancêtres firent l'emblème de la grande Révolution française » (cité dans Martelli et al., 2020 : 58).

Cependant, la position des communistes vis-à-vis de Hugo demeure structurellement ambigüe : s'ils arrachent Hugo au consensus républicain pour le revendiquer comme leur, il n'empêche qu'ils profitent de sa patrimonialisation préalable et du prestige que la République (bourgeoise) s'est attachée à lui conférer. Tout cela, en dernière analyse, fait fond sur une conception dialectique de l'histoire en vertu de laquelle la bourgeoisie, classe dépassée par l'histoire, ne peut plus désormais assumer ses propres grands artistes, forcément visionnaires et en avance sur leur temps (parce que grands, précisément), et nécessairement vus comme des jalons d'une longue " marche au marxisme » (Bernard, 1972 : 233). Lorsque, sur de telles bases, les organisations membres du Front populaire entreprennent en 1935, sous l'impulsion du PC, d'organiser leurs propres commémorations de Hugo (manifestations, récitals, concerts...), il s'agit moins de refuser purement et simplement la logique patrimoniale qui préside à la réception de Hugo que de la déplacer et de la réinventer en célébrant un patrimoine à la fois national et de classe ; le contre-patrimoine communiste, si l'on peut dire, est donc d'autant plus efficace qu'il emprunte au patrimoine national officiel tout en opérant une modification de sens. Bien souvent, donc, la réception militante de Hugo n'est pas exactement l'inverse de sa réception patrimoniale, mais un déplacement de cette dernière.

\section{Hugo en Résistance}

Pendant la Seconde Guerre mondiale, l'opposition entre usage patrimonial et usage politique de la référence hugolienne se trouble. L'urgence de la situation semble à première vue favoriser un usage directement militant et utilitaire de la figure de Hugo, à l'opposé des pratiques que l'on a définies comme patrimoniales : Hugo cesse d'être une grande figure que l'on honore, mais un combattant que l'on convoque à ses côtés. À l'été 1941 (après la rupture du pacte germano-soviétique), le PC sort clandestinement une brochure contenant des extraits des Châtiments et de L'Année terrible, 
accompagnés d'un paratexte qui rapproche le Second Empire d'une part, la guerre franco-allemande de 1870 d'autre part, de l'Occupation (Parti communiste français, 1941 : 3). Il s'agit alors de ranimer une flamme patriotique en abolissant symboliquement toute distance avec cette grande figure nationale du siècle précédent qu'est Hugo ${ }^{6}$. Mais l'année suivante, dans une livraison de la collection « Les Cahiers du Rhône » intitulée Génies de France (Raymond, 1942 : 161-189, l'entreprise de Marcel Raymond 7 consiste au contraire à faire fond sur la distance, à assumer l'extranéité de Hugo : celui-ci, exilé dans son siècle comme il l'était dans son île, parle à ces nouveaux exilés que sont les résistants en ébranlant les coordonnées oppressives du présent). Ce que Raymond réalise ici est bien une sorte de patrimonialisation, en tout cas de muséification symbolique des grandes gloires littéraires françaises : parmi les " génies de France », Hugo voisine avec Agrippa d'Aubigné, Montesquieu, Baudelaire ou Bergson. Même si en l'occurrence la célébration de Hugo est le fait d'un individu et non des pouvoirs publics, elle est tout de même adossée à une collection littéraire emblématique de la Résistance et prend de ce fait une dimension collective ; et surtout, elle vient précisément révéler la défaillance des autorités publiques compromises avec l'occupant et incapables d'entretenir le patrimoine national.

Le geste de Raymond prend place dans une époque où l'existence même de la France est menacée, raison pour laquelle la réactivation mémorielle et patrimoniale a une fonction immédiatement politique. L'hommage aux poètes et aux écrivains sert à attester la grandeur de la patrie en danger ; c'est de cette manière que James Steel (1991 : 35) analyse la stratégie de Vercors consistant à faire énumérer par l'officier allemand von Ebrennac, dans Le Silence de la mer (1942), la liste des auteurs français dont il voit les livres sur les rayonnages d'une bibliothèque (Vercors, $1945: 35$ ) : pour von Ebrennac, il s'agit de plaider en faveur d'une collaboration culturelle francoallemande ; pour Vercors, il s'agit de rappeler aux Français qui le liront qu'ils ont un patrimoine à défendre. Hugo figure bien entendu dans la liste, très accueillante du reste puisqu'elle comprend 21 autres noms. Mais si Hugo se fond là dans un ensemble d'auteurs dont beaucoup n'appartiennent pas à une tradition spécifiquement militante (Baudelaire, Fénelon, Flaubert...), il jouit aussi bien souvent, chez d'autres auteurs de la Résistance, d'un privilège tenant à son statut de poète combattant, de poète adversaire de l'oppression (du Second Empire en particulier). Dans l'avant-propos que Paul Éluard donne anonymement au recueil collectif L'Honneur des poètes en 1943, Hugo est ainsi l'un des quatre représentants (avec Walt Whitman, Rimbaud et Maïakowski) d'une poésie militante, à la hauteur des circonstances ([Éluard], 1943 : 9). Les coordonnées particulières de la période 1940-1944 autorisent une conjonction en fait assez exceptionnelle entre la logique patrimoniale et la logique militante - la convocation du patrimoine étant, vu les circonstances, ipso facto militante.

\section{Conclusion}

Schématiquement, on peut donc opposer une approche patrimoniale d'une part, et une approche politique et militante d'autre part, de la figure de Hugo. Dans le premier cas, il s'agirait de faire fond sur un consensus plus ou moins fantasmé, ou de chercher à l'établir de manière volontariste ; dans le second cas, il s'agirait au contraire de l'arracher à ce consensus national. Mais il est encore plus intéressant de considérer les circonstances dans lesquelles cette opposition schématique se trouble ou se nuance. À certains égards, la patrimonialisation de Hugo est un fait établi, et on ne peut repolitiser Hugo (comme le fait notamment le PC en 1935) qu'en déplaçant à son profit, bien plutôt qu'en annulant purement et simplement, ce statut patrimonial. Ainsi, pour arracher Hugo à un consensus républicain teinté de conservatisme, il faut redéfinir à nouveaux frais une sorte de consensus (tout aussi voire davantage fantasmé que le précédent) permettant une sorte de re-patrimonialisation partisane. Il s'agit, au fond, d'un cas typique de lutte pour l'hégémonie culturelle. Dans ce cas, le moment de la politisation s'appuie sur, et incorpore dialectiquement, le moment de la patrimonialisation. Inversement, lorsque les hommes qui célèbrent la création du cours 
« Victor Hugo » doivent se replier sur une position défensive, cela signifie en fait qu'ils sont contraints par leurs adversaires d'extrême droite de reconnaître que la patrimonialisation de Hugo est, elle-même, un projet politique ; que le consensus patrimonial autour de lui est plus fantasmé que réel ; et, en définitive, que ni la République ni ses symboles ne sont si bien installés qu'ils ne le croient. Dans ce cas, c'est la patrimonialisation qui se révèle in fine politique. Les choses à cet égard deviendront claires après 1940, quand effectivement la célébration patrimoniale et le geste de résistance ne feront qu'un.

\section{Bibliographie}

Les articles extraits du dossier de presse La Chaire de Victor Hugo à la Sorbonne : Article [sic] de presse 1925-1935 (BnF, cote 4-RF-28305) sont suivis d'un astérisque. La pagination des articles a disparu et n'est donc pas indiquée.

Antoine (Gérald). 2002. " Hugo-Claudel : duo-duel ». Année Victor Hugo, revue internationale d'études hugoliennes, 1, p. 233-244.

Aragon (Louis). 1935. « L'actualité de Victor Hugo ». L'Humanité, 29 mai, p. 1-2.

Aragon (Louis). 2007. Euvres poétiques complètes, vol. 1. Paris : Gallimard (Bibliothèque de la Pléiade).

Arthuis (Gaston). 1926. « On inaugure aujourd'hui la chaire Victor Hugo ». Comoedia, 2 février*.

Babilas (Wolfgang). 2002. Études sur Louis Aragon, vol. 2. Münster : Nodus Publikationen (Münstersche Beitrage zur Romanischen Philologie).

Barbarant (Olivier). 2002. «Avez-vous lu Hugaragon? ». La Lecture littéraire, numéro spécial, février, p. 137-147.

Bernard (Jean-Pierre). 1972. Le Parti communiste français et la question littéraire. Grenoble : PUG.

Bonnard (Abel). 1926. « Victor Hugo ». Les Débats, 27 février*.

Bouju (Marie-Hélène). 2010. Lire en communiste : Les maisons d'édition du Parti communiste français, 1920-1968. Rennes : PUR (Histoire).

DOI : 10.400o/books.pur.105650

Brahamcha-Marin (Jordi). 2017. «"Ceux qui pieusement sont morts pour la patrie..." : la poésie des monuments aux morts ». Çédille: revista de estudios franceses, 13, p. 83-95.

Brahamcha-Marin (Jordi). 2018. La Réception critique de la poésie de Victor Hugo en France (1914-1944). Thèse de doctorat, Le Mans Université.

Céard (Henry). 1922. "Chaire Victor Hugo ». Le Petit Marseillais, 3 décembre*.

Chaline (Jean-Pierre). 2007. « De la reconstruction aux années 1960 », p. 67-88 in La Sorbonne au service des humanités : 750 ans de création et de transmission du savoir (1257-2007) / sous la direction de Jean-Robert Pitte. Paris : Presses de l'université Paris-Sorbonne.

Claudel (Paul). 1965. Euvres en prose. Paris : Gallimard (Bibliothèque de la Pléiade).

Daudet (Léon). 1926. « La confusion du baroque et du sublime ». L'Action française, 18 février*.

Denizot (Nathalie). 2015. « Patrimonialisation de la littérature (xixe siècle - xxe siècle), p. 109121 in Les Patrimoines littéraires à l'école : Tensions et débats actuels / sous la direction de Marie-France Bishop et Anissa Belhadjin. Paris : Honoré Champion (Didactique des lettres et des cultures).

Duguet (Camille). 1915. « Causerie féminine ». Le Journal, 13 décembre, p. 5.

[Éluard (Paul)]. 1943. «L'honneur des poètes », avant-propos à L'Honneur des poètes. Paris : Minuit.

Fréville (Jean). 1935. « La révolution vue par Victor Hugo ». L'Humanité, 27 mai, p. 6.

Hargrove (June). 1997. «Les statues de Paris », p. 1855-1886 in Les Lieux de mémoire, vol. 2 / sous la direction de Pierre Nora. Paris : Gallimard (Quarto).

Houdart-Mérot (Violaine). 2015. « Le patrimoine littéraire dans le secondaire en France : une histoire fluctuante et politique ", p. 31-44 in Les Patrimoines littéraires à l'école. Tensions et débats actuels / sous la direction de Marie-France Bishop et Anissa Belhadjin. Paris : Honoré Champion (Didactique des lettres et des cultures).

L’Humanité. 1935. « Le gouvernement célèbre chichement la mort de Victor Hugo ». 23 mai, p. 1 et 3 .

Journal officiel de la République française. Débats parlementaires. Chambre des députés. 1916. 15 septembre [séance du 14 septembre]. 
Journal officiel de la République française. Débats parlementaires. Chambre des députés. 1919. 17 avril [séance du 16 avril].

Journal officiel de la République française. Débats parlementaires. Chambre des députés. 1922. 8 novembre [séance du 7 novembre].

Journal officiel de la République française. Débats parlementaires. Chambre des députés. 1924. 15 novembre [séance du 14 novembre].

Lafargue (Paul). 1979. La Légende de Victor Hugo [1885]. Texte présenté par Tivadar Gorilovics. Debrecen : Kossuth Lajos Tudományegyetem (Studia Romanica, Series litteraria, 6).

Lermina (Jules). 1930. « Le martyre d’Étienne Dolet ». Le Populaire, 19 août, p. 4.

Martinet (Chantal). 1985. «Les hommages publics », p. 257-298 in La Gloire de Victor Hugo / sous la direction de Pierre Georgel. Paris : Éd. de la Réunion des musées nationaux.

Martelli (Roger), Vigreux (Jean) \& Wolikow (Serge). 2020. Le Parti rouge : Une histoire du PCF, 1920-2020. Paris : Armand Colin (Mnémosya).

Millet (Claude). 2002-2003. « Actualité de Victor Hugo : réflexion sur le succès du bicentenaire de 2002 ». Revista da Universidade de Aveiro, 19-20, p. 7-17.

Nizan (Paul). 1935. « Hugo et nous ». L'Humanité, 2 juin, p. 4.

Nora (Pierre). 2011. Présent, nation, mémoire. Paris : Gallimard (Bibliothèque des histoires).

Nozière ([Fernand]). 1926. « L'hommage au Poète ». L'Avenir, 2 février*.

Ory (Pascal). 1994. La Belle Illusion : Culture et politique sous le signe du Front populaire, 19351938. Paris : Plon (Civilisations et mentalités).

Parti communiste français. 1941. « Note de l'éditeur » in Victor Hugo, Les Châtiments, L’Année terrible (extraits). Paris : Imprimerie spéciale du Parti communiste français.

Le Petit Parisien. 1915. Entrefilet sans titre. 18 mars, p. 2.

Le Petit Parisien. 1935. « Une manifestation d'amitié franco-yougoslave ». 26 octobre, p. 7.

Prévotat (Jacques). 2004. L'Action française. Paris : PUF (Que sais-je ?).

DOI : 10.3917/puf.prevo.2004.01

Prost (Antoine). 1997. « Les monuments aux morts : culte républicain ? Culte civique ? Culte patriotique ? ", p. 199-223 in Les Lieux de mémoire, vol. 1 / sous la direction de Pierre Nora. Paris : Gallimard (Quarto).

Raymond (Marcel). 1942. Génies de France. Neuchâtel : La Baconnière (Les Cahiers du Rhône, 4).

Richepin (Jean). 1914. "Inauguration de la statue de Victor Hugo à Guernesey ». Site de l'Académie française : http://www.academie-francaise.fr/inauguration-de-la-statue-de-victorhugo-guernesey [consulté le 12 juillet 2021].

Souday (Paul). 1926a. «Un accès d'hugophobie ». Le Temps, 12 février*.

Souday (Paul). 1926b. Article sans titre. Le Temps, 18 février*.

Steel (James). 1991. Littératures de l'ombre : Récits et nouvelles de la Résistance (1940-1944). Paris : Presses de la Fondation nationale des sciences politiques.

Le Surréalisme au service de la Révolution. 1933. 15 mai, 6. [Reproduit en fac-similé : Le Surréalisme au service de la Révolution. 1976. Paris : J.-M. Place.]

Le Temps. 1922. Article sans titre, 29 décembre*.

Tuc (Pierre). 1926. «Victor Hugo ». L'Action française, 4 mars*.

Vercors. 1945. Le Silence de la mer. Paris : Minuit.

Zyromski (Ernest). 1925. " À propos de la Chaire Victor-Hugo. La conclusion d'un débat ». Le Figaro, 22 décembre, p. 1.

\section{Notes}

1 Les spécialistes d'Aragon ne sont pas d'accord sur la date d'écriture exacte du poème, ni donc sur la question de savoir si le poème fait ou non allusion au déboulonnage de la statue par les Allemands. Olivier Barbarant (2002 : 142) pense que oui, Wolfgang Babilas (2002 : 590-591) estime que non.

2 Cette référence aux enfants «très habillés » s'éclaire si l'on sait que, dans la statue en question, Hugo est représenté nu.

3 L'histoire de ce cours "Victor Hugo » peut être reconstituée, au moins jusqu'au milieu des années trente, grâce aux articles réunis dans le dossier de presse La Chaire de Victor Hugo à la Sorbonne : Article [sic] de presse 1925-1935 (BnF, cote 4-RF-28305). Les références à ces articles seront suivies d'un astérisque. Les articles ayant été découpés, les numéros des pages ne figurent pas dans le dossier de presse. Nous ne pouvons donc les indiquer. 
4 Rappelons qu'à l'époque, les radicaux ne sont pas encore membres du Front populaire ; ils ne s'y associeront, à l'initiative du PC, qu'en juillet 1935. Le Congrès mondial des écrivains de juin 1935 est l'un des événements qui préparent cet élargissement de l'alliance.

5 La ligne de Paul Lafargue, hostile à Hugo, est dès lors complètement abandonnée après avoir été un temps défendue par certains intellectuels du parti (Lafargue, 1979: 37-41).

6 La rupture du pacte germano-soviétique détermine le PC à s'engager dans la republication de textes littéraires relatifs à la guerre de 1870. Outre L'Année terrible de Hugo, donc, le parti édite également, en août 1941, et de manière tout aussi clandestine, Le Père Milon de Maupassant (Bouju, 2010 : 172).

7 Marcel Raymond (1897-1981) est un critique littéraire suisse, professeur à l'université de Genève, et l'un des principaux représentants (avec Albert Béguin) de l' « école de Genève ». Les Cahiers du Rhône sont un périodique suisse, emblématique de la résistance intellectuelle chrétienne, dirigé par Albert Béguin.

\title{
Pour citer cet article
}

Référence papier

Jordi Brahamcha-Marin, « Victor Hugo entre réception patrimoniale et réception politique (19021944) », Culture \& Musées, 38 | 2021, 29-50.

Référence électronique

Jordi Brahamcha-Marin, « Victor Hugo entre réception patrimoniale et réception politique (19021944) », Culture \& Musées [En ligne], 38 | 2021, mis en ligne le 10 novembre 2021, consulté le 08 décembre 2021. URL : http://journals.openedition.org/culturemusees/6592 ; DOI : https://doi.org/10.4000/culturemusees.6592

\begin{abstract}
Auteur
Jordi Brahamcha-Marin

Le Mans Université

Jordi Brahamcha-Marin est normalien, agrégé de lettres modernes, docteur en langue et littérature française. Actuellement ATER à l'université du Mans, il participe aux travaux du Groupe Hugo (Université de Paris) et du projet interdisciplinaire HumaRom (« Humanités romantiques »). Ses recherches concernent l'œuvre de Victor Hugo et sa réception, ainsi que les rapports entre la littérature et la Commune. Il a soutenu sa thèse de doctorat en 2018 à l'université du Mans : La Réception critique de la poésie de Victor Hugo en France (1914-1944), sous la direction de Franck Laurent. II a publié " "Ceux qui pieusement sont morts pour la patrie..." : la poésie des monuments aux morts » (dans Çédille: revista de estudios franceses, avril 2017, $n^{\circ} 13$ ) et « Victor Hugo dans la Grande Guerre » (dans Dix-neuf: Journal of the Society of Dix-Neuviémistes, 2017, vol. 21, $n^{\circ} 1$ ). II est l'auteur, avec Alice de Charentenay, d'une anthologie intitulée La Commune des écrivains : Paris 1871, vivre et écrire l'insurrection, parue chez Gallimard (Folio classique) en 2021.

Courriel : Jordi.brahamcha[at]univ-lemans.fr
\end{abstract}

\section{Droits d'auteur}

Culture \& Musées 